\title{
1 Outcome of patients with advanced ovarian cancer who do not 2 undergo debulking surgery: A single institution retrospective review
}

3

Claudia Marchetti ${ }^{\mathrm{a}, \mathrm{b}}$, Rebecca Kristeleit ${ }^{\mathrm{a}}$, Mary McCormack ${ }^{\mathrm{c}}$, Tim Mould ${ }^{\mathrm{d}}$, Adeola Olaitan $^{\mathrm{d}}$, Martin Widschwendter ${ }^{\mathrm{d}}$, Nicola MacDonald ${ }^{\mathrm{d}}$, Jonathan A Ledermann ${ }^{\mathrm{a}}$.

${ }^{a}$ UCL Cancer Institute and UCL Hospitals, London, UK

${ }^{\mathrm{b}}$ Department of Gynecological and Obstetrical Sciences and Urological Sciences, University "Sapienza", Rome, Italy

${ }^{\mathrm{c} C a n c e r}$ Division, University College Hospital, UCL Hospitals, London, UK

${ }^{\mathrm{d}}$ Departments of Gynaecological Oncology, Women's Health University College Hospital, UCL Hospitals, London, UK

*corresponding author

Correspondence to:

Jonathan A Ledermann,

UCL Cancer Institute and UCL Hospitals, 90 Tottenham Court Rd., London W1T 4TJ, United Kingdom

Phone: 44-20-7679-9874

Fax number: 44-20-7679-9899

E-mail: j.ledermann@ucl.ac.uk 


\section{Abstract}

41 OBJECTIVE: To assess the outcome of patients with advanced ovarian cancer (OC) who were

42 treated without surgery, having received upfront chemotherapy and no interval debulking surgery

43 (IDS).

44 METHODS: Retrospective analysis of medical and chemotherapy records of consecutive patients

45 with OC between 2005 and 2013 at UCL Hospitals London, UK who received neoadjuvant

46 chemotherapy (NACT) and were then found to be unsuitable for IDS following review by the

47 multidisciplinary team.

48 RESULTS: Eighty-three patients (18\%) out of 467 receiving NACT did not undergo IDS. Median 49 age was 70 years (range 33-88); 51.8\% presented with stage IV disease. Forty-three patients 50 received carboplatin and paclitaxel (CP) (51.8\%) and 37 received carboplatin alone (C) (44.6\%); 3

$51(3.6 \%)$ patients received other platinum-based combinations. Reasons for not proceeding to surgery 52 were: poor response to chemotherapy after 3-4 cycles of NACT $(61 / 83,73.5 \%)$; comorbidities $53(12 / 83,14.5 \%)$; patient decision $(4 / 83,4.8 \%)$. Six patients $(7.2 \%)$ received $<3$ cycles of NACT due 54 to a worsening clinical condition. The median overall survival (OS) for patients not undergoing IDS 55 was 18 months (95\% CI 10-20 months). Forty-four (53\%) patients received $>2$ lines of 56 chemotherapy. In a univariate analysis $\mathrm{CP}$, age $<70$ years, and absence of comorbidities were 57 factors influencing OS. In a multivariate analysis only having received CP remained independently 58 associated with OS (HR 0.49, 95\% CI 0.29-0.84).

59 CONCLUSIONS Chemotherapy alone can provide reasonable disease control in patients unsuitable 60 for IDS and CP should be used if possible. 
62 Key words: advanced ovarian cancer, surgery, chemotherapy, neoadjuvant, debulking, carboplatin,

63 paclitaxel

64

65

66

67

68

69

70

71

72

73

74 INTRODUCTION

75 Epithelial Ovarian Carcinoma (EOC) is the leading cause of death from gynecological cancer in the

76 Western World. For women presenting with advanced disease the 5-year survival rate is

77 approximately $30 \%[1]$. Survival of women with epithelial ovarian cancer has improved partly as a

78 consequence of more aggressive surgery to achieve optimal cytoreduction, the use of platinum-

79 based treatment and better treatment of recurrent disease [2]. Nonetheless, approximately $80 \%$ of

80 patients who present with advanced disease develop progression or relapse and die within 5 years

81 from diagnosis[3].

82 Optimal primary debulking surgery followed by platinum-based chemotherapy [3] is the

83 recommended treatment for advanced ovarian cancer (FIGO III-IV). Neoadjuvant chemotherapy

84 (NACT) followed by interval debulking surgery (IDS) can be considered an alternative first-line

85 treatment for patients in whom primary cytoreductive surgery is not possible or contraindicated due

86 to co-morbidity [4-6]. Recent studies have shown similar outcome to primary surgery when interval 
87 debulking surgery (IDS) is performed after three cycles of neoadjuvant chemotherapy followed by

88 three post-IDS cycles of chemotherapy [4-6].

89 It has been estimated that in 10-25\% [6-8] of patients surgical debulking may be not feasible even

90 after NACT, due to poor response to chemotherapy, poor or worsening of performance status,

91 significant co-morbdities, or patients desire to avoid extensive surgery that might require bowel

92 resection.

93 For these women chemotherapy is the primary treatment. It is usually given with palliative intent but

94 little is known about the outcome of these patients

95 The aim of this retrospective study was to understand the natural history of patients with advanced

96 stages of EOC, treated with chemotherapy alone.

97

\section{MATERIALS AND METHODS}

99 All women with a diagnosis of invasive EOC who were treated between January 2005 and 100 December 2013 at UCL Hospitals, London UK were included in this audit. Data were collected 101 between October and November 2014 by reviewing the medical records, radiological imaging, 102 chemotherapy prescriptions and outcome information.

103 The inclusion criteria were as follows: (1) histologically confirmed diagnosis of epithelial ovarian

104 cancer; (2) not suitable for primary or interval debulking surgery; (3) having received primary 105 chemotherapy and (4) availability of medical records.

106 Staging was performed radiologically and defined in accordance with the FIGO (International 107 Federation of Gynecology and Obstetrics) classification for ovarian cancer. All patients had 108 previously undergone histological review by a specialist in gynaecological pathology. Patients with 109 a borderline tumor or a non-epithelial tumor were excluded.

110 All patients were treated with platinum-based chemotherapy and underwent radiological evaluation

111 after 3 or 4 cycles of chemotherapy. They were assessed for surgery by the Multidisciplinary Team. 
112 Criteria for a poor response and consequently unsuitability for surgery were defined as follows:

113 diffuse deep infiltration of the root of the small bowel mesentery, widespread bowel serosal

114 involvement, multiple parenchymatous liver metastases, infiltration of the duodenum and/or

115 pancreas and/or the large vessels of the hepatic-duodenal ligament, celiac trunk or behind the porta

116 hepatis, multiple lung metastases.

117 The medical charts were reviewed to obtain information on the reason for not undergoing surgery,

118 the type of first line chemotherapy, dates of treatment and the reasons for dose reductions and

119 delays. The Charlson Comorbidity index (CCI) score [9] was used retrospectively to assess co-

120 morbidity.

121 Response was assessed by physical examination, serial measurement of CA125, and computed 122 tomographic imaging. Response at the end of treatment was assessed by CA125 according to GCIG 123 criteria [10] and radiological assessment (computed tomographic scan). Progression was defined by 124 clinical or radiological findings and the time to progression was taken as the date of radiological 125 evidence of progression. Further treatments were recorded and overall survival was calculated from 126 the date of primary diagnosis to date of death or to last follow-up visit for the patients still alive. 127 Median follow-up period was measured from the date of primary diagnosis to the time of last 128 follow-up visit.

129 Chi-square or Fisher's exact test was used for comparison of categorical variables. A logistic 130 regression model was applied to determine the effect of independent variables (age, grading, 131 presence of comorbidities (CCI)/ pulmonary embolism, stage, and histology) on the choice of 132 chemotherapy. Survival was calculated using the Kaplan-Meier method. Log-rank test was used to 133 compare survival between groups. Multivariate analysis for prognostic factors was performed by 134 Cox's proportional hazards regression model. All P values were two-sided, and the p-value was set 135 at 0.05. All statistical calculations were carried out using SPSS for Mac version 22.0 (SPSS Inc., 136 USA). 


\section{RESULTS}

141 During the study period primary chemotherapy was given to 467 patients with ovarian cancer and 83

142 patients (18\%) did not proceed to surgery, and are the subject of this study.

143 The median age was 70 years (range 33-88 years). Two age categories were defined: 70 years old or 144 younger, and greater than 70 years old: the median age was 61 years (range 33-70) in the former, 145 and 79 years (range 71-88) in the latter. Clinical and pathological characteristics of patients are 146 described in table 1 . Ten patients $(12 \%)$ had previous history of other cancers. Patients in the older 147 group were more frequently affected by comorbidities (according to $\mathrm{CCI}$ ), $65.9 \%$ compared to $14845.2 \%$ in the younger patients; Forty-three patients $(51.8 \%)$ had stage IV disease and 10 patients 149 (19.3\%) presented with a pulmonary embolism (PE), or developed a PE during chemotherapy (5 150 patients).

151 Paclitaxel and carboplatin were given to 43 patients $(51.8 \%)$ and 37 received carboplatin alone 152 (44.6\%); three patients (3.6\%) received other platinum -based combinations. The median number of 153 cycles given was 6 (range 1-8), and 24\% of patients received less than 6 cycles. Five patients also 154 received bevacizumab (6.3\%). Patients older than 70 years (OR 0.31, CI95\% 0.10-0.93, $\mathrm{p}=0.007$ ) 155 and those presenting with at least one comorbidity (OR 0.31, CI95\% 0.10-0.90, p=0.016) were 156 more likely to receive carboplatin alone treatment rather than carboplatin plus paclitaxel.

157 Six patients $(7.2 \%)$ received less than 3 cycles of chemotherapy, stopping because of a worsening 158 clinical condition, and were therefore not assessable for IDS (table 2).

159 Sixty-one patients $(73.5 \%)$ out of the whole group were judged to be unsuitable for optimal surgical 160 debulking on the basis of a poor response to chemotherapy. Other reasons for having not having 161 surgery were patient decision $(4 / 83,4.8 \%)$ and the presence of comorbidities in $12 / 83,14.5 \%)$. The 
162 comorbidities were severe cardiovascular disease (CVD) (7 patients), a cerebrovascular accident

163 (CVA) (1 patient) and significant worsening pulmonary embolus (8 patients), including 4 patients

164 with CVD or CVA.

165 At the end of chemotherapy 53 patients (63.8\%) had a partial response on CT imaging, $12(14.4 \%)$

166 had stable disease and ten (12\%) patients had disease progression. In 2 patients radiological

167 information was absent $(2.4 \%)$ and 6 patients were not assessable for IDS, as stated above.

168 According to CGIG criteria, among the 59 patients whose CA125 measurements were available and

169 evaluable, $50(84.7 \%)$ had a response, including $17(28.8 \%)$ with a complete response, whilst there

170 were $6(10.1 \%)$ who did not achieve any response and 3 were not evaluable (CA 125 below normal

171 range at diagnosis).

172 Thirty-nine out of 83 patients (46.9\%) received only one line of chemotherapy; 24 (28.9\%) patients 173 received a second line of chemotherapy following disease progression. Subsequently, 15 patients $174(18 \%)$ received 3 lines, 2 patients (2.4\%) received 4 lines, 1 patient (1.2\%) received 5 lines and 2 175 patients (2.4\%) received 6 lines of chemotherapy. Overall, $44(53 \%)$ patients received $>2$ lines of 176 chemotherapy.

177 The median follow-up period was 18 months. The median OS of the overall population was 18 178 months (95\% CI 10-20 months).

179 Analysing OS according to type of chemotherapy received in the overall population (Fig. 1), women 180 who underwent carboplatin plus paclitaxel had better median OS of 27 (95\% CI 20-33 months) 181 months compared with 15 (95\% CI 14-19 months) months for patients who received carboplatin 182 alone $(\log$ rank: $\mathrm{p}=0.002 ;$ HR $0.45,95 \%$ CI $0.27-0.75)$

183 In a univariate analysis (table 3), type of chemotherapy (carboplatin vs. carboplatin plus paclitaxel) 184 and age ( $>$ or $\leq 70$ years), and absence of comorbidities were factors influencing OS. However, in 185 the multivariate analysis (table 3 ) only treatment with the combination of carboplatin plus paclitaxel 186 was independently associated with OS (log rank: $p=0.002$; HR 0.49, 95\% CI 0.29-0.84). 


\section{DISCUSSION}

190 Debulking surgery to remove all residual disease remains the cornerstone of ovarian cancer 191 treatment [11]. Nonetheless, even in clinical trials of NACT in patients with potentially 192 operable disease, $10-25 \%$ are not able to undergo debulking surgery [6,7]. There is little 193 information about the outcome of this group of women. The key finding was that $18 \%$ of all 194 patients in our institution undergoing primary chemotherapy do not undergo surgery and 195 their median survival was 18 months. A poor response to chemotherapy was the main reason 196 for failure to proceed to surgery and in $27 \%$ the decision was made not to operate because of 197 co-morbidity or patient choice. However, 68.8\% patients achieved a partial response to 198 chemotherapy, 53\% received a further line of chemotherapy, and $24 \%$ had 3 or more lines of 199 treatment.

200 The median age of our population was 70 years, higher than the population median age of EOC at 201 diagnosis [3]. Co-morbidity is more common in older patients so they are more likely to receive 202 single agent carboplatin chemotherapy. Both age $\geq 70$ years and CCI score $\geq 1$ were independent 203 predictors of single agent chemotherapy. This is in accordance with other experiences [12]. 204 Although carboplatin and paclitaxel are considered as standard of treatment for stage II-IV ovarian 205 cancer [13], single agent carboplatin compares well to a carboplatin plus paclitaxel combination [14] 206 and it has been proposed that it is an acceptable standard treatment for older patients [15].

207 We found that receiving the combination of carboplatin and paclitaxel is independently associated 208 with better survival, even after adjusting for age and comorbidities. This underlines the importance 209 of identifying which factors should preclude the use of paclitaxel in elderly patients.

210 Approximately half of our patients received two or more lines of treatment. Whilst surgery plays a 211 key role in the management of ovarian cancer, patients unable to undergo surgery should still be 
212 considered for active management as in some of them, multiple lines of treatment are able to control

213 the disease for many months. In our series, though we did not have information on symptom control

214 or quality of life, the administration of several lines of chemotherapy contributed to the finding of a

215 median OS of 18 months, which compares favourable to other reported series in which the median

216 OS was in the range of 8-11 months [8,14-17] for patients unsuitable for surgery. Shalowitz et al

217 recently reported a shorter OS for those who only received systemic treatment (12 months), and an

218 even shorter OS for those who did not receive any treatment (1.4 months); unfortunately data about

219 treatment administered and number of chemotherapy lines are lacking and further comparisons are

220 not possible. Overall, we might speculate that the availability of different combinations of treatment

221 we described can provide some of these women with the opportunity of extended palliation without

222 surgery as they can receive several lines of treatment in the absence of surgery.

223 The present study was a single institution retrospective investigation. Whilst consecutive patients

224 were included, a selection or referral bias could have occurred, and this might have influenced the

225 analyses, particularly the comparison of single agent and combination therapy. Nonetheless we

226 believe that our findings provide useful and relevant information to decision-making about surgery

227 for clinicians treating patients with neoadjuvant therapy. Cytoreductive surgery remains the

228 cornerstone of treatment of advanced EOC but when it cannot be performed chemotherapy provides

229 good palliation and disease control for many patients.

\section{CONFLICT OF INTEREST}

232 The authors declare that there are no conflicts of interest. 
1. Seidman, J. D., Yemelyanova, A., Cosin, J. a, et al. Survival rates for international federation of gynecology and obstetrics stage III ovarian carcinoma by cell type: a study of 262 unselected patients with uniform pathologic review. Int. J. Gynecol. Cancer 22, 367-71 (2012).

241 2. Vaughan S, Coward JI, Bast RC Jr. et al. Rethinking ovarian cancer: recommendations for improving outcomes. Nature Reviews Cancer 11, 719-725 (2011).

243 3. Jayson, G. C., Kohn, E. C., Kitchener, H. C. \& Ledermann, J. a. Ovarian cancer. Lancet 6736, 244 (2014).

245 4. Kehoe S, Hook J, Nankivell M, et al. Chemotherapy or upfront surgery for newly diagnosed 246 advanced ovarian cancer: Results from the MRC CHORUS trial. J. Clin. Oncol. Vol 31, 5500 (2013).

247 5. da Costa Miranda V, de Souza Fêde ÂB, Dos Anjos CH. et al. Neoadjuvant chemotherapy with six cycles of carboplatin and paclitaxel in advanced ovarian cancer patients unsuitable for primary surgery: Safety and effectiveness. Gynecol. Oncol. 132, 287-291 (2014).

250 6. Vergote I, Tropé CG, Amant F, et al. Neoadjuvant chemotherapy or primary surgery in stage IIIC 251 or IV ovarian cancer. N. Engl. J. Med. 363, 943-953 (2010).

252 7. Pignata S, Scambia G, Katsaros D, et al. Carboplatin plus paclitaxel once a week versus every 3 253 weeks in patients with advanced ovarian cancer (MITO-7): a randomised, multicentre, open-label, phase 3 trial. Lancet. Oncol. 15, 396-405 (2014).

8. Saha A, Varughese M, Gallagher CJ, et al. Primary chemotherapy for inoperable ovarian, fallopian tube, or primary peritoneal cancer with or without delayed debulking surgery. Int. J. Gynecol. Cancer 22, $257 \quad 566-72(2012)$.

258 9. Charlson, M. E., Pompei, P., Ales, K. L. \& MacKenzie, C. R. A new method of classifying 259 prognostic comorbidity in longitudinal studies: development and validation. J. Chronic Dis. 40, 373-83 260 (1987).

261 10. Rustin GJ, Vergote I, Eisenhauer E, et al. Definitions for response and progression in ovarian cancer clinical trials incorporating RECIST 1.1 and CA 125 agreed by the Gynecological Cancer Intergroup (GCIG). Int. J. Gynecol. Cancer 21, 419-23 (2011). 
264 11. Bristow, R. E., Tomacruz, R. S., Armstrong, D. K., et al. Survival effect of maximal cytoreductive 265 surgery for advanced ovarian carcinoma during the platinum era: A meta-analysis. J. Clin. Oncol. 20, $266 \quad 1248-1259(2002)$

267 12. Jørgensen TL, Teiblum S, Paludan M., et al. Significance of age and comorbidity on treatment 268 modality, treatment adherence, and prognosis in elderly ovarian cancer patients. Gynecol. Oncol. 127, 367$269374(2012)$.

270 13. Covens A, Carey M, Bryson P, et al. Systematic review of first-line chemotherapy for newly 271 diagnosed postoperative patients with stage II, III, or IV epithelial ovarian cancer. Gynecol Oncol. 2002 272 Apr;85(1):71-80.

273 14. International Collaborative Ovarian Neoplasm Group. Paclitaxel plus carboplatin versus standard 274 chemotherapy with either single-agent carboplatin or cyclophosphamide, doxorubicin, and cisplatin in 275 women with ovarian cancer: the ICON3 randomised trial. Lancet 360, 505-15 (2002).

276 15. Stoeckle E, Boubli B, Floquet A, et al. Optimal timing of interval debulking surgery in advanced 277 ovarian cancer: Yet to be defined? Eur. J. Obstet. Gynecol. Reprod. Biol. 159, 407-412 (2011).

278 16. Pignata S, Breda E, Scambia G, et al. A phase II study of weekly carboplatin and paclitaxel as first279 line treatment of elderly patients with advanced ovarian cancer. A Multicentre Italian Trial in Ovarian 280 cancer (MITO-5) study. Critical Reviews in Oncology/Hematology 66, 229-236 (2008).

281 17. Shalowitz DI, Epstein AJ, Ko EM, Giuntoli RL 2nd. Non-surgical management ofovarian cancer: 282 Prevalence and implications. Gynecol Oncol. 2016 Jul;142(1):30-7.

\section{Conflict of interest statement}

The authors declare that they have no competing interests. 
291 LEGEND

\section{TABLES}

293 Table 1: Patients Pathological and Clinical characteristics.

294 Table 2: Characteristics of patients receving less than 3 cycles.

295 Table 3: Univariate and multivariate analysis of prognostic factors.

296

297 FIGURE

298 Figure 1 : Overall survival of patients receiving carboplatin alone (37 patients) or carboplatin plus 299 paclitaxel (43 patients) (log rank: $p=0.003)$

300

301

302 